\title{
Primary pancreatic mucosa-associated lymphoid tissue lymphoma transformed into diffuse large B-cell lymphoma: a case report and literature review
}

\author{
Xiaolin $\mathrm{Wu}^{\wedge}$, Guimin Zhao, Haisheng Liu, Yingzhen Yao, Zhe Gao, Qingna Guo \\ Department of Hematology, The Fourth Hospital of Hebei Medical University, Shijiazhuang, China \\ Correspondence to: Qingna Guo. Department of Hematology, The Fourth Hospital of Hebei Medical University, 169 Tianshan Street, Shijiazhuang \\ 050035, China. Email: w112233440804@163.com.
}

\begin{abstract}
Primary pancreatic lymphoma (PPL) is an extremely rare malignant tumor that accounts for less than $2 \%$ of extranodal malignant lymphomas and $0.5 \%$ of pancreatic tumors. The most common symptoms of PPL are abdominal pain (83\%), abdominal mass (58\%), and weight loss (50\%). The final diagnosis of PPL is based on cell histology, and the most common histological subtype is diffuse large B-cell lymphoma (DLBCL). The number of reported cases of primary pancreatic mucosa-associated lymphoid tissue (MALT) lymphoma is limited. The conversion rate of MALT lymphoma varies between $3 \%$ and $12 \%$. However, the transformation of primary pancreatic MALT lymphoma to DLBCL has not been reported. We present a case of a 58-year-old man whose main symptoms were nausea and vomiting. Magnetic resonance imaging of the abdomen showed space-occupying lesions in the neck and body of the pancreas. Histopathological examination after surgical resection was diagnosed as primary pancreatic MALT lymphoma. Local radiotherapy was recommended and rejected by the patient's family. The disease progressed in a short time. Left supraclavicular lymph node resection biopsy and pathological examination showed DLBCL. After the initiation of chemotherapy, glaucoma appeared in the right eye during treatment, and chemotherapy was suspended. The disease progressed rapidly and the patient eventually died.
\end{abstract}

Keywords: Primary pancreatic lymphoma; mucosa-associated lymphoid tissue lymphoma; diffuse large B-cell lymphoma; pathological type; case report

Submitted Aug 06, 2021. Accepted for publication Oct 02, 2021.

doi: 10.21037/apm-21-2418

View this article at: https://dx.doi.org/10.21037/apm-21-2418

\section{Introduction}

Primary pancreatic lymphoma (PPL) is an extremely rare form of pancreatic cancer that often represents a diagnostic and therapeutic challenge due to its scarcity and propensity to be clinically and radiologically mistaken for pancreatic adenocarcinoma. It comprises less than $2 \%$ of extranodal malignant lymphomas and $0.5 \%$ of pancreatic tumors (1-4). The most common symptoms of PPL are abdominal pain $(83 \%)$, abdominal mass (58\%), and weight loss (50\%) (3). Obstructive jaundice can be caused by extrinsic duct compression by tumor and/or nodal metastases and/or tumorous portal vein thrombosis. Therefore, PPL should be considered when radiographic images show lymphangitic lesions around the hilar or pancreas. Additionally, PPL can present as follicular, small lymphocytic, and T-cell lymphoma, The classic symptoms of nodal non-Hodgkin's lymphoma (NHL), such as fever, chills, and night sweats, are uncommon, and are found in only $2 \%$ of patients $(5,6)$.

The definitive diagnosis of PPL is based on cytohistological examination. Alternatively, a laparoscopy or

^ ORCID: 0000-0002-0644-2921. 


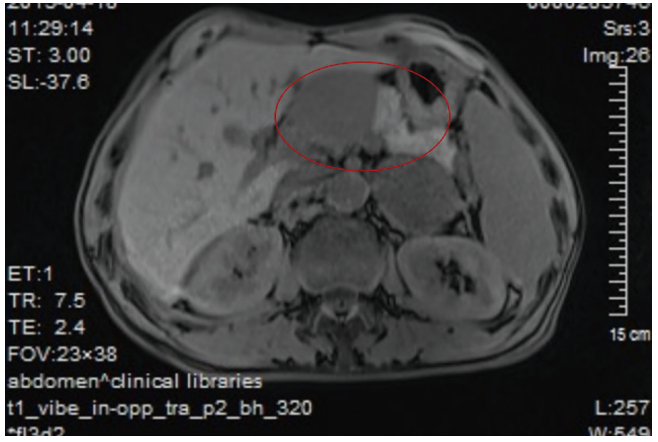

Figure 1 Abdominal magnetic resonance imaging. (The red circle is a pancreatic tumor).

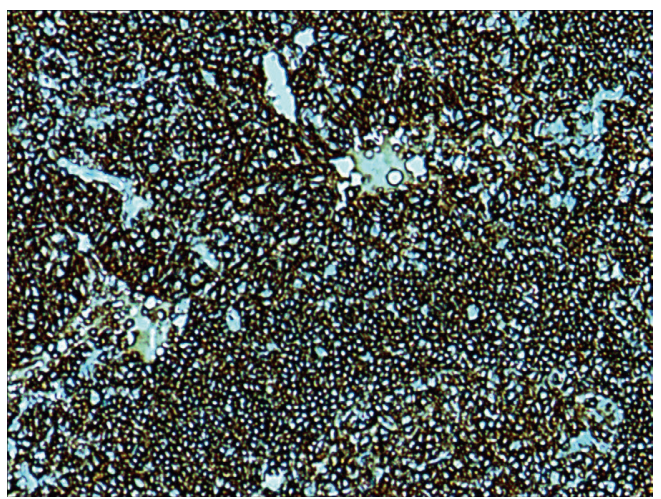

Figure 2 CD20. Streptavidin-horseradish peroxidase staining. Magnification $\times 100$.

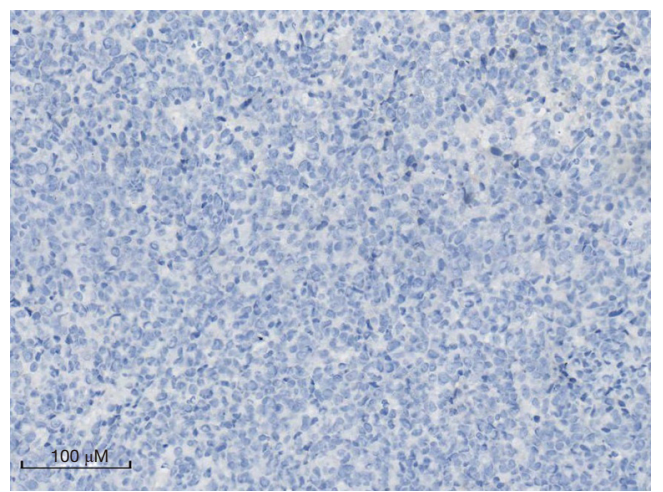

Figure 3 CD23. Streptavidin-horseradish peroxidase staining. Magnification $\times 100$.

laparotomy may be performed to conduct a biopsy of the pancreatic mass or lymph nodes. The most common histological subtype of PPL is diffuse large B-cell lymphoma (DLBCL). To date, a limited number of cases have reported primary mucosa-associated lymphoid tissue (MALT) lymphoma. The transformation rate in MALT lymphoma varies between $3 \%$ and $12 \%$ (7-9). To the best of our knowledge, the transformation of primary pancreatic MALT lymphoma into DLBCL has not yet been reported. We report a case of a 58-year-old man who presented with nausea and vomiting as the main symptoms. The patient was diagnosed with primary pancreatic MALT lymphoma. Transformation of primary MALT lymphoma into DLBCL occurred within a short period of time, and the patient died due to disease progression.

We present the following article in accordance with the CARE reporting checklist (available at https://dx.doi. org/10.21037/apm-21-2418).

\section{Case presentation}

The patient presented with intermittent nausea and vomiting for 4 months. No abnormality was found on gastroscopy. Ultrasonography of the abdomen revealed a pancreatic space-occupying lesion and hypoechogenicity in the liver. Computed tomography (CT) displayed a spaceoccupying lesion in the left inner rear lobe of the liver and in the left adrenal gland. Magnetic resonance imaging (MRI) of the abdomen revealed space-occupying lesions in the neck and body of the pancreas, space-occupying lesions in the left adrenal region, and hepatic hemangioma (Figure 1). Pathological findings were as follows: neurofibroma in the left adrenal gland, extranodal marginal zone lymphoma (MZL) of MALT in the pancreas, as well as detected expression levels of $\mathrm{CD} 20^{+}, \mathrm{CD}^{-}, \mathrm{CD} 79 \mathrm{a}^{+}$, CD45RO- ${ }^{-}, \mathrm{Ki}-67$ (10-60\%), BCL-2 ${ }^{+}, \mathrm{CD}_{10}^{-}, \mathrm{BCL}^{-}{ }^{-}$, CD5-, CD21, CD23, and cyclin $\mathrm{D1}^{-}$(Figures 2-5). The timeline of interventions and outcomes of the case are shown in Figure 6.

The patient did not report fever or night sweats during the course of disease, and he lost $5 \mathrm{~kg}$ within 6 months. The patient's medical and family history were unremarkable.

CT scan of the chest and abdomen showed emphysema and pulmonary bullae in the absence of other abnormalities. No abnormality was detected in bone marrow biopsy. Results of blood routine test were as follows: white blood cells (WBC), $5.85 \times 10^{9} / \mathrm{L}$; neutrophils, $50.5 \%$; lymphocytes, $39.8 \%$; monocytes, $7.8 \%$; eosinophilic, $1.1 \%$; basophilic, $0.8 \%$; red blood cells, $4.35 \times 10^{12} / \mathrm{L}$; hemoglobin, $131.5 \mathrm{~g} / \mathrm{L}$; platelets, $303 \times 10^{9} / \mathrm{L}$; and lactate dehydrogenase, $205 \mathrm{U} / \mathrm{L}$. Antibody test results of hepatitis A, B, and C were negative.

The diagnosis was primary pancreatic MALT lymphoma. 
Because the relevant examinations failed to show that the surgical margin was negative, local radiotherapy was recommended, which was refused by the patient's family members.

At 8 months after discharge, the patient developed swelling and pain in the left back, numbness in the left chest

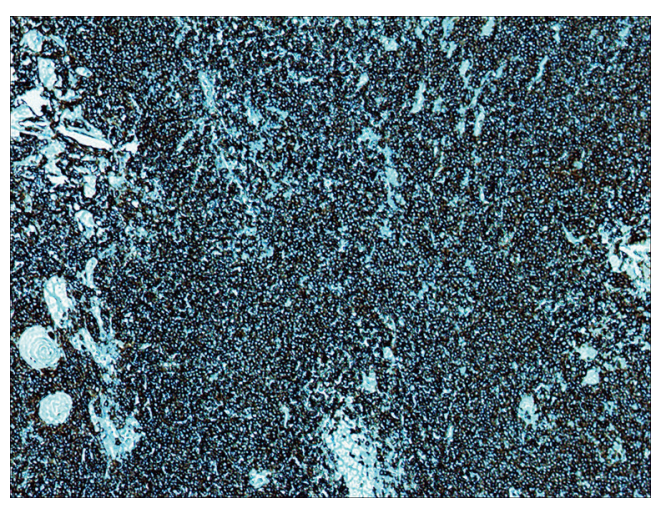

Figure 4 BCL-2. Streptavidin-horseradish peroxidase staining. Magnification $\times 100$.

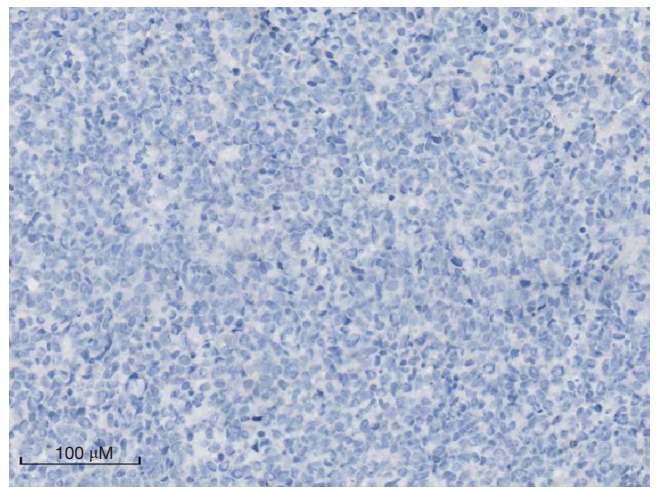

Figure 5 CD10. Streptavidin-horseradish peroxidase staining. Magnification $\times 100$. wall and left upper limb, and a mass on the left clavicle that gradually increased to $10 \times 7 \mathrm{~cm}$ in size. CT examination showed that the lymph nodes beside the left carotid artery, the left posterior cervical triangle, the left supraclavicular lymph nodes, the left armpit, and the bilateral iliac vessels were swollen, which was consistent with NHL manifestations, and pleural thickening and metastasis were clearly observed. Biopsy of the left supraclavicular lymph nodes indicated NHL; therefore, immunohistochemistry was used, and the levels of CD20 $0^{+}, \mathrm{Pax}_{-} 5^{+}$, Ki-67 (80\%), multiple myeloma oncogene $1^{-/+}$, B-cell lymphoma-2 ${ }^{+}$, B-cell lymphoma- $6^{+}$, and $\mathrm{CD} 10^{-}$were determined. Final diagnosis was the conversion of primary pancreatic MALT lymphoma to DLBCL. The patient's disease stage was IVA.

After the initiation of chemotherapy, glaucoma appeared in the right eye during treatment, and chemotherapy was therefore suspended. The lymphoma progressed again, central nervous system infiltration occurred, and the lymphoma progressed to leukemia, which ultimately resulted in death.

All procedures performed in studies involving human participants were in accordance with the ethical standards of the institutional and/or national research committee(s) and with the Helsinki Declaration (as revised in 2013). Written informed consent was obtained from the patient for publication of this case report and accompanying images. A copy of the written consent is available for review by the editorial office of this journal.

\section{Discussion}

The recent World Health Organization classification recognizes 3 subtypes of MZL: extranodal MZL of MALT, splenic MZL, and nodal MZL. The most common subtype of MZL is MALT lymphoma, which accounts for $70 \%$ of all MZL and occurs at different extranodal sites, including the

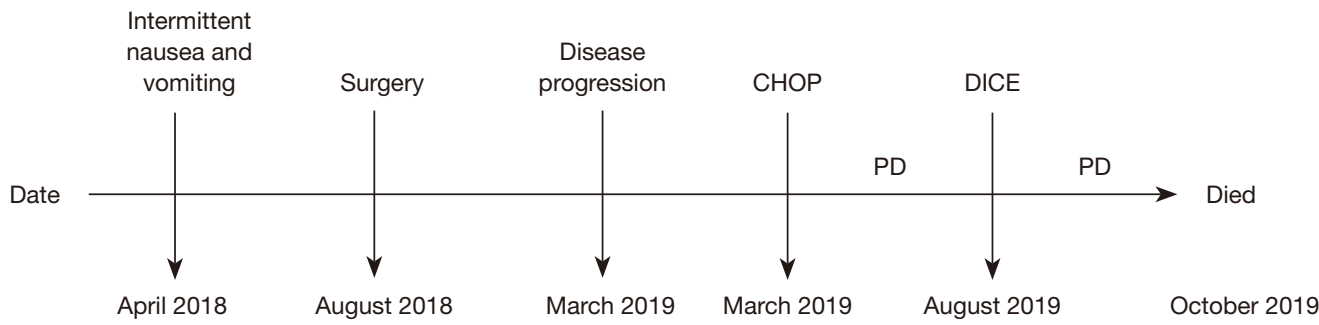

Figure 6 Timeline of interventions and patient outcomes. CHOP, cyclophosphamide, doxorubicin, oncovin, prednisone; DICE, dexamethasone, ifosfamide, cisplatin, etoposide; PD, progressive disease. 
stomach (70\%), lung (14\%), ocular adnexa (12\%), thyroid (4\%), and small intestine (including immunoproliferative small intestinal disease, $1 \%)(10-12)$.

The most common histological type of PPL is NHL. Freeman et al. analyzed 1,467 cases of extranodal lymphoma, and only 9 pancreatic cases were involved, accounting for less than 1\% (13). In another review involving 620 cases of primary gastrointestinal NHL, only $1.2 \%$ originated from the pancreas (14). In the majority of patients with pancreatic cancer, whether adenocarcinoma or lymphoma, salivary gland tumors dominate. PPL mainly occurs in the head of the pancreas, although this tumor can also be found in the body and tail. Pancreatic lymphoma is often described as a large, homogeneous mass with extrapancreatic infiltration, with or without lymph node involvement. Tumors of the body and tail of the pancreas constitute one-third of pancreatic neoplasms. In the present case, MRI of the abdomen revealed space-occupying lesions in the neck and body of the pancreas, space-occupying lesions in the left adrenal region, and hepatic hemangioma. Behrns et al. defined the diagnostic criteria of PPL as follows: mass predominantly located within the pancreas, with grossly involved lymph nodes confined to the peri-pancreatic region, no palpable superficial lymphadenopathy, no hepatic or splenic involvement, no mediastinal nodal enlargement on chest radiography, and normal WBC count (15). The present case was in line with these diagnostic criteria.

Extranodal lymphoma is composed of morphologically heterogeneous small $\mathrm{B}$-cells, including marginal zone (centrocyte-like) cells, monocytoid-like cells, small lymphocytes, and scattered immunoblasts and centroblastlike cells. The B cells of MALT lymphoma share the cytological features and immunophenotype [CD20 $\mathrm{CD} 21^{+}, \mathrm{CD} 35^{+}$, immunoglobulin $\left.(\mathrm{Ig}) \mathrm{M}^{+}, \mathrm{IgD}-\right]$ of marginal zone B cells $(16,17)$. The histological feature of MALT lymphoma comprises infiltration of the marginal zone and spreading diffusely into the surrounding tissue. MALT lymphoma cells share the same cytological and immune phenotypical $\left(\mathrm{CD} 20^{+}, \mathrm{CD} 21^{+}, \mathrm{CD} 35^{+}, \mathrm{IgM}^{+}\right.$, and $\left.\mathrm{IgD}^{-}\right)$ features as marginal zone B cells. It is reported that MALT lymphoma rarely undergoes histological transformation and progresses to high-grade lymphoma, which only occurs in $3-12 \%$ of cases $(7,8)$. MALT lymphoma has been reported to be related to gene abnormalities, such as $\mathrm{p} 16^{\mathrm{INK}}$ and $\mathrm{p} 53$ activation (12). C-MYC overexpression, BCL-6 translocation, and BCL-6 protein expression are also related to histological transformation in MALT lymphoma $(18,19)$.
It has often been detected alongside MALT lymphoma $(70 \%)$. In addition, in the refractory or recurrent stage of MALT lymphoma, biopsy and histological examination are both essential for the detection of the incidence of histological transformation.

The 5 -year survival rate of MALT lymphoma has been reported to be as high as $89 \%$ (20); however, the prognosis of pancreatic malignant lymphoma is poor (21). Takagi et al. reported that the average survival time was 8 months in 25 cases with definite prognosis (22). There are histological types other than MALT lymphoma. Such as DLBCL. It has been suggested that the formation of MALT is mainly caused by infectious diseases, such as Helicobacter pylori, hepatitis virus, and chlamydia, or immune disorders caused by autoimmune diseases $(23,24)$. To our knowledge, MALT lymphoma is a form of lymphoma involving the MALT, and most frequently occurs in the stomach, although any mucosal site can be affected.

Transformation of MALT lymphoma to DLBCL is the result of the emergence of an increased numbers of transformed blast cells. A pathological study of 467 patients with MALT lymphoma showed that the majority of participants (78\%) had localized disease in histological transformation, and the 5-year progression-free survival (PFS) and overall survival rates were favorable in histological transformation patients ( $80 \%$ and $94 \%$, respectively). Histological transformation does not only affect prognosis. A multifactor analysis showed that late-stage histological transformation was a significant disadvantage for posthistological transformation PFS (25). In the present case, disease progression occurred shortly after the diagnosis of primary pancreatic MALT lymphoma, and the pathological type transformed into invasive lymphoma. Genetic tests were not performed due to patient refusal.

The treatment of patients with marginal lymphoma is similar to that of those with follicular lymphoma, and current guidelines recommend the commencement of treatment in patients with a high tumor mutational burden or as soon as symptoms appear. Radiotherapy has been used to effectively treat NHL of both low and high grades in the primary, consolidative, salvage, and palliative settings. For localized disease, systemic treatment is combined with radiotherapy, which in this setting results in improved PFS and reduces disease recurrence at the primary site. For advanced DLBCL, radiotherapy is used in the management of severe disease to improve the control of local disease; for non-gastric MALT, radiation therapy is 
considered for symptomatic situations. Other therapeutic options for ocular adnexal MALT lymphoma include systemic chemotherapy, surgery alone, and a watch-andwait strategy. Pancreatic malignant lymphoma has a poor prognosis, and it has been reported that prognosis can be improved by resection and chemotherapy postoperatively (26-28). Koniaris et al. compared age, sex, histopathological type, and stage of lymphoma in 15 patients with PPL who were treated surgically for stage I or II pancreatic cancer, and found that the complete remission rate was significantly improved in the surgical treatment group (26). If the pathological type converts to DLBCL, a drug combination therapy used to treat NHL (R-CHOP: rituximab; cyclophosphamide; doxorubicin; oncovin; prednisone) is an appropriate therapeutic option. The present case of primary pancreatic MALT lymphoma was stage I, and local radiotherapy was recommended because pathological assessment could not prove a negative margin. However, the patient's family members refused the suggested therapy, and the disease recurred and progressed. Pathological type changed to DLBCL. Chemotherapy for invasive lymphoma with multiple courses and regimens was found ineffective, and the patient eventually died due to disease progression.

\section{Conclusions}

The incidence of primary pancreatic MALT lymphomas is extremely low, and prognosis is satisfactory. However, the pathological type transforms to high-grade during treatment, and prognosis is poor after transformation. In terms of treatment, a retrospective analysis and review of relevant literature showed that surgery could be effective for patients with non-gastrointestinal stage I MALT lymphoma, while combination chemotherapy has been shown to be more effective for patients with non-gastrointestinal stage IV MALT lymphoma and pathological type transformation.

\section{Acknowledgments}

We appreciate the linguistic assistance provided by TopEdit (www.topeditsci.com) during the preparation of this manuscript.

Funding: This work was supported by clinical features and prognostic analysis of lymphoproliferative diseases with hypergammaglobulinemia, Hebei Province, China (No. 20160655).

\section{Footnote}

Reporting Checklist: The authors have completed the CARE reporting checklist. Available at https://dx.doi. org/10.21037/apm-21-2418

Conflicts of Interest: All authors have completed the ICMJE uniform disclosure form (available at https://dx.doi. org/10.21037/apm-21-2418). The authors have no conflicts of interest to declare.

Ethical Statement: The authors are accountable for all aspects of the work in ensuring that questions related to the accuracy or integrity of any part of the work are appropriately investigated and resolved. All procedures performed in studies involving human participants were in accordance with the ethical standards of the institutional and/or national research committee(s) and with the Helsinki Declaration (as revised in 2013). Written informed consent was obtained from the patient for publication of this case report and accompanying images. A copy of the written consent is available for review by the editorial office of this journal.

Open Access Statement: This is an Open Access article distributed in accordance with the Creative Commons Attribution-NonCommercial-NoDerivs 4.0 International License (CC BY-NC-ND 4.0), which permits the noncommercial replication and distribution of the article with the strict proviso that no changes or edits are made and the original work is properly cited (including links to both the formal publication through the relevant DOI and the license). See: https://creativecommons.org/licenses/by-nc-nd/4.0/.

\section{References}

1. Zucca E, Roggero E, Bertoni F, et al. Primary extranodal non-Hodgkin's lymphomas. Part 1: Gastrointestinal, cutaneous and genitourinary lymphomas. Ann Oncol 1997;8:727-37.

2. Lin H, Li SD, Hu XG, et al. Primary pancreatic lymphoma: report of six cases. World J Gastroenterol 2006;12:5064-7.

3. Saif MW. Primary pancreatic lymphomas. JOP 2006;7:262-73.

4. Haji AG, Sharma S, Majeed KA, et al. Primary pancreatic lymphoma: Report of three cases with review of literature. Indian J Med Paediatr Oncol 2009;30:20-3. 
5. Nishimura R, Takakuwa T, Hoshida Y, et al. Primary pancreatic lymphoma: clinicopathological analysis of 19 cases from Japan and review of the literature. Oncology 2001;60:322-9.

6. Arcari A, Anselmi E, Bernuzzi P, et al. Primary pancreatic lymphoma. Report of five cases. Haematologica 2005;90:ECR09.

7. Conconi A, Franceschetti S, Aprile von Hohenstaufen $\mathrm{K}$, et al. Histologic transformation in marginal zone lymphomas. Ann Oncol 2015;26:2329-35.

8. Zucca E, Conconi A, Laszlo D, et al. Addition of rituximab to chlorambucil produces superior event-free survival in the treatment of patients with extranodal marginalzone B-cell lymphoma: 5-year analysis of the IELSG-19 Randomized Study. J Clin Oncol 2013;31:565-72.

9. Isaacson P, Wright DH. Malignant lymphoma of mucosaassociated lymphoid tissue. A distinctive type of B-cell lymphoma. Cancer 1983;52:1410-6.

10. Swerdlow, SH; Campo, E; Pileri, SA, et al. The 2016 revision of the World Health Organization classification of lymphoid neoplasms. Blood 2016;127:2375-90.

11. Radaszkiewicz T, Dragosics B, Bauer P. Gastrointestinal malignant lymphomas of the mucosa-associated lymphoid tissue: factors relevant to prognosis. Gastroenterology 1992;102:1628-38.

12. Thieblemont C, Berger F, Dumontet C, et al. Mucosaassociated lymphoid tissue lymphoma is a disseminated disease in one third of 158 patients analyzed. Blood 2000;95:802-6. Erratum in Blood 2000;95:2481.

13. Freeman C, Berg JW, Cutler SJ. Occurrence and prognosis of extranodal lymphomas. Cancer 1972;29:252-60.

14. Lal A. Miscellaneous: 620 primary gastrointestinal nonHodgkins lymphomas Va retrospective review. Ann Occup Hyg 2005;16:c218.

15. Behrns KE, Sarr MG, Strickler JG. Pancreatic lymphoma: is it a surgical disease? Pancreas 1994;9:662-7.

16. Tsuboi $\mathrm{K}$, Iida S, Inagaki H, et al. MUM1/IRF4 expression as a frequent event in mature lymphoid malignancies. Leukemia 2000;14:449-56.

17. Isaacson PG, Chott A, Nakamura S, et al. Extranodal marginal zone lymphoma of mucosa-associated lymphoid tissue (MALT lymphoma). WHO classification of tumours of haematopoietic and lymphoid tissues 2008:214-7.

18. Huang W, Guo L, Liu H, et al. C-MYC overexpression predicts aggressive transformation and a poor outcome in mucosa-associated lymphoid tissue lymphomas. Int J Clin Exp Pathol 2014;7:5634-44.
19. Flossbach L, Antoneag E, Buck M, et al. BCL6 gene rearrangement and protein expression are associated with large cell presentation of extranodal marginal zone B-cell lymphoma of mucosa-associated lymphoid tissue. Int J Cancer 2011;129:70-7.

20. Olszewski AJ, Castillo JJ. Survival of patients with marginal zone lymphoma: analysis of the Surveillance, Epidemiology, and End Results database. Cancer 2013;119:629-38.

21. Kobashi K, Matsuda T, Takakura N, et al. A case of pancreatic malignant lymphoma. Journal of the Japanese Society of Clinical Surgeons 1998;59;2157-60.

22. Takagi T, Nishikawa H, Hisaka A, et al. An autopsy case of pancreatic malignant lymphoma that developed during the course of chronic hepatitis C. Journal of the Japanese Society of Gastroenterology 2004;101:1227-31.

23. Shaye OS, Levine AM. Marginal zone lymphoma. J Natl Compr Canc Netw 2006;4:311-8.

24. Kojima M, Nakamura S, Futamura N, et al. Malignant lymphoma in patients with rheumatic diseases other than Sjögren's syndrome: a clinicopathologic study of five cases and a review of the Japanese literature. Jpn J Clin Oncol 1997;27:84-90.

25. Maeshima AM, Taniguchi H, Toyoda K, et al. Clinicopathological features of histological transformation from extranodal marginal zone B-cell lymphoma of mucosa-associated lymphoid tissue to diffuse large B-cell lymphoma: an analysis of 467 patients. Br J Haematol 2016;174:923-31.

26. Koniaris LG, Lillemoe KD, Yeo CJ, et al. Is there a role for surgical resection in the treatment of early-stage pancreatic lymphoma? J Am Coll Surg 2000;190:319-30.

27. Battula N, Srinivasan P, Prachalias A, et al. Primary pancreatic lymphoma: diagnostic and therapeutic dilemma. Pancreas 2006;33:192-4.

28. Du X, Zhao Y, Zhang T, et al. Primary pancreatic lymphoma: a clinical quandary of diagnosis and treatment. Pancreas 2011;40:30-6.

Cite this article as: $\mathrm{Wu} \mathrm{X}$, Zhao G, Liu H, Yao Y, Gao Z, Guo Q. Primary pancreatic mucosa-associated lymphoid tissue lymphoma transformed into diffuse large B-cell lymphoma: a case report and literature review. Ann Palliat Med 2021;10(10):11226-11231. doi: 10.21037/apm-21-2418 